which had been incubated in serum pre-treated with the enzyme IdeS (Immunoglobulin-G degrading enzyme of S. pyogenes, which selectively cleaves IgG). Complement deposition and neutrophil phagocytosis of unencapsulated $S$ pneumoniae TIGR4 strain was reduced in IdeS treated serum compared to untreated serum. These data demonstrate that there are naturally acquired functionally significant antibody responses to a range of conserved $S$ pneumoniae protein antigens. The same antigens induce responses in diverse populations, suggesting that these protein antigens would be useful components for a polyvalent protein vaccine that is broadly protective against $S$ pneumoniae infections.

\section{S128 HIGHLY INVASIVE CAPSULAR SEROTYPES OF STREPTOCOCCUS PNEUMONIAE BIND HIGH LEVELS OF FACTOR H AND ARE RESISTANT TO COMPLEMENT AND PHAGOCYTOSIS}

doi:10.1136/thoraxjnl-2011-201054b.128

${ }^{1} \mathrm{C}$ Hyams, ${ }^{2} \mathrm{~K}$ Trzcinski, ${ }^{3} \mathrm{D} \mathrm{M}$ Weinberger, ${ }^{4} \mathrm{M}$ Lipsitch, ${ }^{1} \mathrm{~J} \mathrm{~S}$ Brown. ${ }^{1}$ Centre for Respiratory Research, UCL, London, UK; ${ }^{2}$ Department of Pediatric Immunology and Infectious Disease, University Medical Centre Utrecht, Utrecht, Netherlands; ${ }^{3}$ Division of International Epidemiology and Population Studies, National Institute of Health, Bethesda, Maryland, USA; ${ }^{4}$ Department of Epidemiology, Epidemiology and Department of Immunology and Infectious Diseases, Harvard School of Public Health, Boston, USA

The Streptococcus pneumoniae polysaccharide capsule is an essential virulence factor that varies in structure between serotypes. While certain serotypes are highly invasive, it is unknown why these serotypes frequently cause infection yet others generally cause nasopharyngeal colonisation. Complement is vital in immunity to pneumococcus, and the capsule is known to affect complement deposition. Activation of the alternative complement pathway is promoted by factor $\mathrm{B}(\mathrm{Bf})$ binding, but inhibited by factor $\mathrm{H}(\mathrm{FH})$ activation. We hypothesised that capsule effects on $\mathrm{FH}$ and $\mathrm{Bf}$ interactions could alter $S$ pneumoniae complement sensitivity, partially explaining serotype-dependent differences in invasiveness. $\mathrm{C} 3 \mathrm{~b} / \mathrm{iC} 3 \mathrm{~b}$ deposition, $\mathrm{FH}$ and $\mathrm{Bf}$ binding to $S$ pneumoniae were measured using flow cytometry assays on 20 distinct capsule-switch variants constructed on TIGR4 genetic background. These strains were therefore identical in protein structure, differing only in capsular serotype. Phagocytosis was investigated using an established flow cytometry assay, fresh human neutrophils and FAMSE labelled $S$ pneumoniae. $\mathrm{FH}$ binding showed wide variations between TIGR4-capsular switched strains, with a 7.5-fold difference between the highest (serotype2) and lowest (serotype11A) results. Differences in FH binding between strains did not correlate with capsular thickness, or with capsule structural motifs such as numbers of carbon atoms per repeating unit. FH binding negatively correlated with $\mathrm{Bf}$ binding and $\mathrm{C} 3 \mathrm{~b} / \mathrm{iC} 3 \mathrm{~b}$ deposition, demonstrating that increased $\mathrm{FH}$ binding was associated with reduced alternative pathway activity and increased resistance to complement. IgG binding did not correlate with $\mathrm{C} 3 \mathrm{~b} / \mathrm{iC} 3 \mathrm{~b}$ results, suggesting $\mathrm{C} 3 \mathrm{~b}$ deposition was independent of antibody-mediated complement activity. Neutrophil association correlated with $\mathrm{C} 3 \mathrm{~b} / \mathrm{iC} 3 \mathrm{~b}$ deposition $\left(R^{2}=0.47, p=0.0008\right)$ but negatively with $F H$ binding $\left(R^{2}=0.74\right.$, $\mathrm{p}<0.0001$ ), confirming that high $\mathrm{FH}$ binding by pneumococcus was associated with reduced neutrophil phagocytosis. Weakly- and highly-invasive serotypes showed large significant differences between median C3b deposition $(p=0.007)$, neutrophil association $(p=0.0002)$ and FH binding $(p=0.0005)$. Weakly-invasive serotypes had reduced $\mathrm{FH}$ binding, increased $\mathrm{C} 3 \mathrm{~b} / \mathrm{iC} 3 \mathrm{~b}$ deposition and increased neutrophil association. Our novel finding that the degree of FH binding to $S$ pneumoniae capsular serotypes is associated with large variations in virulence offers a mechanistic explanation as to why certain serotypes are more invasive than others. It also provides a potential in vitro method for identifying highly invasive strains.

\section{S129 NEUTROPHIL FUNCTION AND ADVANCING AGE: THE EFFECTS OF SIMVASTATIN IN HEALTH AND DURING PNEUMONIA}

doi:10.1136/thoraxjnl-2011-201054b.129

${ }^{1} \mathrm{E}$ Sapey, ${ }^{1} \mathrm{H}$ Greenwood, ${ }^{1} \mathrm{~J}$ Hazeldine, ${ }^{1} \mathrm{G}$ Walton, ${ }^{1} \mathrm{D}$ Thickett, ${ }^{1} \mathrm{~J} \mathrm{M}$ Lord, ${ }^{2} \mathrm{R}$ A Stockley. ${ }^{1}$ University of Birmingham, Birmingham, UK; ${ }^{2}$ University Hospital Birmingham NHS Foundation Trust, Birmingham, UK

Background Age is associated with a decline in immunity, including neutrophil function. This may partially explain the worsening clinical outcomes seen following pneumonia in the elderly. Statins may improve outcomes from pneumonia although it is unknown whether they influence neutrophil function at conventional therapeutic concentrations. This is crucial, as statins may be beneficial adjuvants during infections.

Methods We studied the effect of $5 \mathrm{ng} / \mathrm{ml}$ Simvastatin (equivalent to $80 \mathrm{mg}$ orally) on key neutrophil functions: speed and accuracy of migration using time-lapse imaging $(\mu \mathrm{m} / \mathrm{min})$, Neutrophil Extracellular Trap formation using cell-impermeable DNA-binding dye (in AFU) and generation of reactive oxygen species (ROS, in RLU).

Results We studied 70 healthy subjects (aged 20-90 years, 10 in each decennial) and 6 young ( $<35$ years) and 6 older ( $>65$ years) patients during an admission with pneumonia. All studied neutrophil functions declined with age (eg, neutrophil chemotaxis, $\mathrm{r}=-0.7, \mathrm{p}<0.001)$. Specifically, average neutrophil chemotaxis for subjects $>65 \mathrm{yrs}$ was $0.72 \mu \mathrm{m} / \mathrm{min}$ ( $\mathrm{SD} \pm 0.28, \mathrm{p}<0.001$ ) slower than subjects $<35$. These neutrophils produced less NETS (average difference $=-1725 \mathrm{AFU} \pm 283, \mathrm{p}=0.007$ ) and peak ROS (average difference $-117 R L U \pm 31 ; p=0.04$ ). Neutrophils from young patients with pneumonia displayed an up-regulation of function that was not seen in older pneumonia patients. There were no ageassociated differences in the surface expression of chemo-attractant receptors, but there appears to be differential intracellular signalling with reduced expression of adhesion molecules. Incubating neutrophils from older subjects with Simvastatin improved all functions back to that seen in young subjects (chemotactic speed $+0.92 \mu \mathrm{m} /$ min $\pm 0.27, p=0.001$ : NET $+1386 \mathrm{AFU} \pm 273, p=0.04$ : ROS $+223 R L U \pm 39, p=0.005)$. Similar improvements were seen with neutrophils from older subjects with pneumonia. This was a dosedependent phenomenon; not seen at higher concentrations of Simvastatin.

Conclusion With age, there is a global deterioration in neutrophil function and no up-regulation when pneumonia is present, which may partially explain the age-associated mortality. Simvastatin upregulates neutrophil function in the elderly, even during pneumonia. This up-regulation may explain the beneficial effects seen clinically. In vivo studies are warranted, to determine if simvastatin should be utilised during episodes of acute infection.

\section{S130 HIV-1 INFECTION OF MACROPHAGES DYSREGULATES PRO- INFLAMMATORY HOST RESPONSES TO MYCOBACTERIUM TUBERCULOSIS THROUGH INHIBITION OF INTERLEUKIN 10}

doi:10.1136/thoraxjnl-2011-201054b.130

${ }^{1} \mathrm{G}$ S Tomlinson, ${ }^{2} \mathrm{P}$ T G Elkington, ${ }^{1} \mathrm{~L}$ Bell, ${ }^{2} \mathrm{~N}$ F Walker, ${ }^{1} \mathrm{~J}$ Tsang, ${ }^{1} \mathrm{~J}$ Brown, ${ }^{1} \mathrm{R}$ Breen, ${ }^{1} \mathrm{M}$ Lipman, ${ }^{1} \mathrm{D}$ R Katz, ${ }^{1} \mathrm{R}$ F Miller, ${ }^{1} \mathrm{~B}$ M Chain, ${ }^{1} \mathrm{M}$ Noursadeghi. ${ }^{1}$ University College London, London, UK; ${ }^{2}$ Imperial College London, London, UK

Introduction Human immunodeficiency virus (HIV)-1 greatly increases the risk of active Mycobacterium tuberculosis (Mtb) 
infection. Both pathogens target macrophages by evading innate immune recognition or intracellular killing. Since macrophages play a key role in inflammatory responses and their resolution, we tested the hypothesis that HIV-1 infection of macrophages may modulate inflammatory responses to co-infection with $\mathrm{Mtb}$ contributing to the immunopathogenesis of active tuberculosis (TB).

Methods Innate immune responses to Mtb were assessed in human macrophages with and without productive HIV-1 infection using genome-wide transcriptional profiling. Array data were validated by quantitative PCR and correlated with protein secretion in cell culture supernatants. The effects of Mtb co-infection on HIV replication were assessed by ELISA. The mechanisms underlying the observed phenotype were examined by Western immunoblotting and using selective inhibitors of innate immune signalling pathways.

Results HIV-1 infection of monocyte-derived macrophages leads to sustained, exaggerated pro-inflammatory responses to $\mathrm{Mtb}$ coinfection, including cytokines and chemokines that may recruit and activate further inflammatory leucocytes, and matrix metalloproteinases which play a role in tissue destruction. This phenotype is associated with rescue of HIV-1 replication following early repression in response to $\mathrm{Mtb}$. Our data suggest that augmented inflammatory responses to $\mathrm{Mtb}$ result from deficient induction of anti-inflammatory interleukin-10 in HIV-1 infected cells. None of these changes were evident in HIV-1 infected macrophages coinfected with Streptococcus pneumoniae, and the specificity of the effect in Mtb co-infection was mirrored by lower IL-10 and higher pro-inflammatory IL-1 $\beta$ in respiratory samples from HIV-1 infected patients with pulmonary TB compared to non-tuberculous respiratory infection. Complementation of deficient IL-10 responses to $\mathrm{Mtb}$ in HIV-1 infected macrophages reverses the exaggerated proinflammatory phenotype. HIV-1 infection attenuates phosphorylation of p38 and ERK1/2 in mitogen activated kinase pathways involved in IL-10 induction downstream of TLR2 and dectin-1 receptor stimulation. IL-10 production in HIV-1 infected cells is also inhibited in response to zymosan stimulation of these pathways. Inhibition of virus maturation with HIV-1 protease inhibitors, does not affect attenuation of IL-10 responses.

Conclusions Deficient induction of homeostatic IL-10 and consequent augmentation of pro-inflammatory responses may contribute to the immunopathogenesis of active $\mathrm{TB}$ and propagation of virus infection in HIV-1/Mtb co-infection.

\section{S131 HUMAN MACROPHAGE MODEL OF BIOMASS SMOKE EXPOSURE SHOWS IMPAIRED INGESTION OF STREPTOCOCCUS PNEUMONIAE}

doi:10.1136/thoraxjnl-2011-201054b.131

${ }^{1}$ A N Aljurayyan, ${ }^{2} \mathrm{D}$ G Fullerton, ${ }^{3} \mathrm{~S}$ Barrett, ${ }^{1} \mathrm{~S}$ B Gordon. ' Liverpool School of Tropical Medicine, Liverpool, UK; ${ }^{2}$ University Hospital Aintree, Liverpool, UK; ${ }^{3}$ University of Liverpool, Liverpool, UK

Background Three billion people worldwide, primarily in less economically developed countries use biomass fuel (BMF) as their main source of household energy. In children, the risk of pneumonia is increased by exposure to BMF smoke by a factor of 1.8. In adults, an epidemiological association between acute pneumonia and outdoor air pollution and tobacco smoke exposure has been demonstrated but there are no data confirming an association between pneumonia and BMF smoke exposure. Few mechanistic studies have been performed in humans investigating the association between BMF exposure and $S$ pneumoniae. This work aimed to assess the phagocytic ability of particulate matter (PM) challenged macrophages on fluorescent labelled beads and $S$ pneumoniae.

Methods In order to model BMF exposure, monocyte derived macrophages (MDMs), from 12 day old buffy coats, were challenged with different types and doses of PM (fine carbon black (FCB), Malawi wood and Norwegian wood). The percentage area of the macrophage cytoplasm that PM occupied (PM load) was calculated using image analysis software (Image SXM). The ability of PM challenged MDMs to phagocytose fluorescent labelled beads and $S$ pneumoniae was assessed by counting the beads / bacteria associated with MDMs, using fluorescent microscopy.

Results Increasing the PM dose was associated with a decrease in the percentage of cells associated with $S$ pneumoniae (Abstract S131 figure 1A) and beads. The same result was observed when the average number of $S$ pneumoniae (or beads) within cells was used as the outcome measure. With all three PMs used, a lower PM load was correlated with a higher capacity to phagocytose $S$ pneumoniae and beads. Malawi wood and Norwegian wood impaired MDMs phagocytic ability more than FCB (Abstract S131 figure 1B).
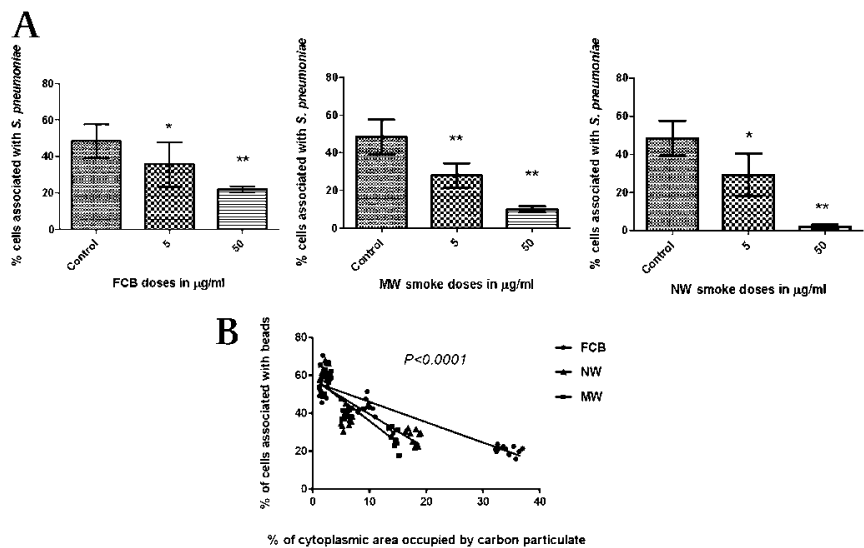

Abstract S131 Figure 1 (A) Comparison of percentage of cells associated with $S$ pneumoniae with 3 different types of particulate matter. (B) Comparison between the percentages of cells associated with $S$ pneumoniae and cytoplasmic area occupied by PM and three different types of PM (FCP, Norwegian wood and Malawi wood).

Conclusions Our data demonstrate that MDMs exposed to PM have impaired ability to phagocytose beads and $S$ pneumoniae and that wood smoke exposed MDMs had a greater phagocytic impairment than FCB. These observations support an association between BMF smoke exposure and pneumonia. Our model enables further work to be carried out on the dose-response of smoke exposure and pneumococcal disease as well as into the pathogenesis of increased susceptibility to pneumococcal infection in BMF exposed individuals.

\section{S132 INVESTIGATING THE ROLE OF PELLIN01, AN E3 UBIQUITIN LIGASE, IN MODULATING SIGNALLING PATHWAYS CONTROLLING THE INFLAMMATORY RESPONSE}

doi:10.1136/thoraxjnl-2011-201054b.132

J A Bennett, L R Prince, C A Stokes, L C Parker, M K Whyte, I Sabroe. University of Sheffield, Sheffield, UK

Viruses, such as Rhinovirus, are a major cause of asthma exacerbations. Pellino1 was identified as an interleukin 1 receptor associated kinase binding partner and has shown to act as an E3ubiquitin ligase that is involved in Lysine-63 polyubiquitination of interleukin 1 receptor associated kinase $1 / 4$ and RIP1, which are key mediators of the Toll-like receptor and interleukin 1 receptor (IL-1R) signalling pathways. As such, these pathways are implicated in responses to both bacterial and viral infections. However, the functional importance of Pellino proteins in regulating immune responses and their role in airway inflammatory diseases is yet to be 